\title{
Laterality of fetal self-touch in relation to maternal stress
}

Nadja Reissland, Senior Lecturer and Deputy Head of Science (Postgrad), Department of Psychology, Durham University, UK *

Ezra Aydin, Research Assistant, Department of Psychology, Durham University, UK

Brian Francis, Professor of Social Statistics, Department of Maths and Statistics, Lancaster University, UK

Kendra Exley, Lead Sonographer, James Cook University Hospital, Middlesbrough, UK.

\section{Shortened form of title}

Fetal laterality and maternal stress

*Correspondence addressed to :

Dr. N. Reissland

Department of Psychology,

Science Site,

Durham University

Durham DH1 3LE, UK

Email: n.n.reissland@durham.ac.uk

Tel : +44 (0)1913343287

Fax: +44 (0)191334 3241 


\begin{abstract}
This longitudinal observational study investigated whether fetuses change their hand preference with gestational age, and also examined the effects of maternal stress on lateralized fetal self-touch. Following ethical approval, fifteen healthy fetuses (8 girls and 7 boys), were scanned four times from 24-36 weeks gestation. Self-touch behaviours which resulted in a touch of the fetal face/head were coded in 60 scans for 10 minutes and analysed in terms of frequency of the fetuses using left and right hands to touch their face. The joint effects of fetal age, stress and sex on laterality were assessed. We modelled the proportion of right self-touches for each fetal scan using a generalised linear mixed model, taking account of the repeated measures design. There was substantial variability in hand preference between fetuses. However, there was no significant increase in the proportion of right-handed touches with fetal age. No sex differences in handedness were identified. However, maternally reported stress level was significantly positively related to fetal left handed self-touches (odds-ratio $0.915 ; \mathrm{p}<0.0001$ ). This longitudinal study provides important new insights into the effect of recent maternal stress on fetal predominant hand use during self-touch.
\end{abstract}


Fetal laterality and maternal stress

Keywords:

Fetus; self-touch; laterality, handedness, maternal stress; fetal age 


\section{Introduction}

Given the importance of lateralized behaviours in development, a number of studies have investigated lateral behavioural biases prenatally with some researchers reporting lateralized behaviours and others not finding any. A number of studies have examined lateralized behaviours by analyzing reaching and whereas others reported frequency of movements and yet others analysed touch.

In contrast to many human and animal studies examining goal directed behaviour such as reaching, in fetal research lateralized touch relates either to self-touch including thumb sucking or to the frequency of arm movements irrespective of touch. Specifically, selftouch or intra-active touch is defined as the simultaneous process of one part of the body involved in the activity of touching and another body part being touched (Yang, Han, Chui, Shen, \& Wu, 2012). Concerning self-touch, Hepper, Shahidullah \& White (1991) using real time sonographic examinations of 274 fetuses, observed for each fetus one instance of thumb sucking. They did not report details of the ultrasound device. Based on one observation of thumb suck per fetus, they found a right thumb preference.

Furthermore, analysing movement frequency, rather than self-touch, Hepper, McCartney, Shannon (1998), using 2-D scans, found that at 10 weeks gestation, 54 fetuses (out of 72) exhibited a greater number of right arm compared with left arm movements. In contrast, Sparling, van Tol, \& Chescheir (1999) videotaped 2-D images of fetal scans projected on a monitor, ranging in age from 14 weeks gestation to term. These researchers coded left and right arm movements and did not detect any differences in the frequency of right or left movements. Ververs, van Gelder-Hasker, de Vries, Hopkins \& van Geijn, 1998) videotaped 2-D scans of 10 fetuses from 12-38 weeks gestation every 4 weeks. Scoring the movements of one fetal arm every 30 seconds they did not find any significant differences 
between frequencies of right or left arm movements. De Vries, Wimmer, Ververs, Hopkins, Savelsbergh \& van Geijn (2001) recorded arm movements of 10 fetuses mid-sagitally in 2-D scans from 12 to 38 weeks gestation, observing them every 4 weeks. They did not find a lateralized preference in terms of frequency of arm movements observed.

In summary, a comparison of self-touch by analysing fetal thumb sucking resulted in some researchers finding differences in prenatal laterality while others did not. For example, the study by Hepper et. al.(1991) in which 274 out of 282 fetuses were observed in real time sucking their thumb, found a right asymmetry. This is in contrast to De Vries et. al. (2001), who found no lateral bias when coding thumb sucking, observed in 4 out of 77 recordings of 10 fetuses.

Similar to studies analysing fetal self-touch with some evidence of lateralized behaviour, some studies on frequency of fetal arm movements do not report any evidence of lateralized movements whereas others do find lateralized behaviour. Specifically, Fong, Buis, Savelsbergh, \& de Vries, (2005) found no difference between flexion of right and left arms in fetuses in cephalic and breech presentation. Myowa-Yamakoshi \& Takeshita (2006), using 4-D ultrasonography to examine arm and hand movements in second to third-trimester fetuses, found no difference between frequency of right and left movements.

Given that self-touch in general is a mode of self-regulation of mild stress (e.g. Landau, Shusel, Eshel, Ben-Aaron, 2003) and furthermore that atypical laterality, i.e., less asymmetry or atypical brain lateralization and non-right-handedness, is associated with stress in adults (e.g. Dragovic \& Hammond, 2005; Heim \& Keil, 2004), the present study investigated fetal lateralized self-touch in relation to maternal stress.

In sum, the objective of this study is to examined longitudinally the relationship between the development of hand laterality from 24 to36 weeks gestation in relation to maternal stress in 
order to establish whether we can observe changes in hand dominance in fetal self-touch and whether it is influenced by maternal self-reported stress during pregnancy. Furthermore, given that some research (e.g.Glover, O'Connor, Heron, Golding, \& ALSPAC Study team, 2004) suggests that males and females are differentially affected by prenatal stress, we tested for sex differences in lateralized touch.

\section{Methods}

\section{Objectives}

There were two principal objectives. First, to investigate fetal lateralized touch behaviour and changes in such behaviour from the second to the third trimester in a longitudinal study, and second, to examine whether maternal stress and fetal sex might be associated with fetal hand preference in touching the face.

\section{Participants}

Fifteen healthy fetuses, 8 girls and 7 boys, were scanned in the second and third trimester. The fetuses were observed four times, resulting in 60 scans, in the mornings in the radiography department where mothers had previously undergone their routine 12 and 20 week medical scans. The scans took place with the mothers lying in a darkened room on their back or on their side depending on the position of the fetus and how comfortable mothers were. The first research scan was performed at a mean age of 24.2 weeks (range 23.9-24.5 weeks); the second at 28.0 weeks (range 27.8-28.2 weeks); the third at 32.1 weeks (range 31.8-32.4 weeks); the fourth at 36.1 weeks (range 36.0- 36.4 weeks). All participants were first time mothers with mean age 27 years (range 19 - 40 years), specifically recruited through the midwives of the antenatal unit of the James Cook University Hospital, 
Middlesbrough, UK. All fetuses were assessed to be healthy after birth (mean: 40 weeks with range $37-42$ weeks gestational age) by a paediatrician, with mean weight 3283 grams (std. dev. 489 grams). Apgar scores were measured at 1 minute (mean 9.06, range 9-10) and 5 minutes (mean: 9.33, range: 9-10). Mothers were self-declared to be non-smoking and not drinking alcohol. Parental handedness was not recorded as previous work suggests that association between fetal handedness and parental handedness is weak (McManus \& Bryden, 1991)

\section{Ethics}

Ethical permission for the study was granted by the County Durham and Tees Valley 2 Research Ethics Committee (REC Ref: 08/H0908/31) and the research and development department of James Cook University Hospital, as well as the Durham University (Department of Psychology ethics committee). All mothers gave informed written consent.

\section{Procedure}

Mothers, who had completed normal 20-week anomaly scans, were invited to participate in this study by the sonographer. All participating mothers received four additional scans at 24, 28, 32 and 36 weeks gestational age, with each mother being scanned in the morning for approximately 15-20 minutes. During consent and before each procedure, mothers were made aware that these additional scans were for research purposes and not routine medical scans. Mothers were provided with a DVD copy of their scans. The fetal face and upper torso were visualized both by means of 4-D colour full frontal or facial profile ultrasound recordings, as well as sequences of traditional 2-D monochrome images. Both 
were recorded for off line analysis with a GE Voluson E8 Expert Ultrasound System using a GE RAB4-8L Macro 4D Convex Array Transducer. Data were recorded directly with the Voluson E8 which has a build in recorder onto DVDs. The scanner hard drive permits only relatively short sections to be recorded and stored; hence this was not an option. The recorded DVDs were imported into the OBSERVER software and coded frame by frame.

Mothers were asked to fill out the Perceived Stress Scale questionnaire to assess their stress levels at each scan. The Perceived Stress Scale (PSS) is a widely used valid and reliable 10 item five-point Likert-based scale (ranging from $0=$ ‘no stress’ experienced during the last month to 4=`very often’ stressed (Cohen, Kamarck, \& Mermelstein, 1983; Cohen \& Williamson,1988) which measures the degree to which mothers perceive their life as stressful. The theoretical maximum of the scale is 40 .

\section{Method of coding fetal movement}

In contrast to previous studies on fetal laterality (Ververs, van Gelder-Hasker, de Vries, Hopkins \& van Geijn 1998; Hepper, Shahidullah \& White 1991), our coding scheme used continuous recording of fetal movements. Ten minutes of segments of the 60 scans, in which the fetal face as well as a hand were visible was selected and coded frame by frame and analysed in terms of frequency of occurrence of self-touch. The offline coding was done by one trained coder who had learned the coding system which we have used previously. A second coder also trained in the coding system and blind to the hypothesis performed the reliability coding.

Coding focused on fetal head and face touches with the left and right arms, hands and fingers of the fetus. Thus, only those touch behaviours which resulted in a touch of the face and head were coded. If a fetus touched the head with an arm this would only be coded if a 
reference point, namely digit, thumb or elbow were visible in order to ensure that laterality could be established. If both hands were visible and touching they were both coded. In the instance a touch was observed this would be marked as the 'beginning of a touch'. The coder observed frame by frame until the hand moved from the face recording this as the 'end' of the touch. Such an observation would count as one touch. Additionally it was agreed that if the same touch behaviour occurred within 5 seconds of a touch being judged to have stopped the second touch would not be included in the frequency count.

However, seeing both hands was not a condition of coding. This conservative coding scheme included the following conditions: either 'little finger' or 'thumb' needed to be visible in order to distinguish right or left hands or if fingers could not be discerned but there was a "visible touch" (verified in 2D and 4D images) and "an elbow" could be seen then this would be used to distinguish right and left hands. Figure 1 illustrates the use of fingers to distinguish the left hand touching the upper face region.

The availability of both 2D and 4D images meant that we were able to minimise as far as possible bias caused by touching movements out of sight of the sampled area.

\section{Insert Figure 1}

\section{Establishing laterality}

For each observation period, the clearest 10 minutes of the scan (i.e. the face was not facing away, placenta was not blocking view of the face) was selected. The frequency of left and right facial touch behaviours was then counted. Additionally, within each scan, the fetus was identified as left handed if the majority of self-touches were with the left hand or arm, 
equal if the fetus used an equal number of left and right touches and right -handed when the majority of self- touches occurred with the right hand.

\section{Reliability}

Using Cohen’s Kappa, reliability was established for 34\% of these scans, which were coded independently by a coder blind to the hypothesis and trained in the coding scheme. This resulted in Cohen's Kappa $=0.965$ (individual scan mean $=0.98$, range 0.97-0.99) in relation to fetal left and right self-touches. Intra-rater reliability was conducted separately from inter-rater reliability. The main coder recoded $18 \%$ of the scans which resulted in Cohens Kappa $=0.974$.

\section{Statistical Analysis}

Assessment of trend in maternal stress over gestational age controlling for fetal sex was carried out through a linear mixed (or multi-level) model (Snijders \& Bosker, 2012) of stress score on linear gestational age and sex, assuming underlying normality of the stress scores, and with two levels of variability - between mothers and within mothers over gestational age. Effects were reported as regression coefficients with 95\% confidence intervals. Formally we can write the model as

$$
\begin{gathered}
\text { stress }_{\mathrm{it}}=\beta_{0}+\mathrm{u}_{\mathrm{i}}+\beta_{1} \text { age }_{\mathrm{it}}+\beta_{2} \text { gender }_{\mathrm{i}}+\varepsilon_{\mathrm{it}} \\
\varepsilon_{\mathrm{it}} \sim \operatorname{Normal}\left(0, \sigma_{\mathrm{e}}^{2}\right) ; \quad \mathrm{u}_{\mathrm{i}} \sim \operatorname{Normal}\left(0, \sigma_{\text {fetus }}^{2}\right)
\end{gathered}
$$

Variability in laterality was described by looking at the handedness of the fetus over gestational age, and examining the laterality switches of each fetus. 
We tested the variability of fetal laterality over gestational age, looking at the number of right handed head touches out of the total number of head touches within each scan. Gestational age trend for both of these was assessed by fitting a binomial logit mixed model to these proportions, with (continuous) age as explanatory variable. The mixed model takes account both of the repeated measures nature of the design, and the response type of the data and accounts for the two levels of variation in the data - between fetus and within fetus. Formally the model can be written as

$$
\operatorname{logit}\left(\mathrm{p}_{\mathrm{it}}\right)=\beta_{0}+\mathrm{u}_{\mathrm{i}}+\beta_{1} \text { age }_{\mathrm{it}} \quad \mathrm{R}_{\mathrm{it}} \sim \operatorname{Binomial}\left(\mathrm{p}_{\mathrm{it}}, \mathrm{N}_{\mathrm{it}}\right)
$$

where $p_{i t}$ is the proportion of right-handed touches $\mathrm{R}_{\mathrm{it}}$ out of all head touches $N_{i t}$ for fetus $i$ at age $t$, and $u_{i}$ is the individual fetus random effect, which is assumed to be normally distributed with mean 0 and variance $\sigma_{\text {fetus }}^{2}$. Significance of each effect in this model was assessed by examining the change in deviance (minus twice the log-likelihood) caused by omitting the effect from the model - this has a chi-squared distribution on $1 \mathrm{df}$ under the null hypothesis. Effects were reported as odds-ratios with 95\% confidence intervals.

Finally, the multivariate joint effects of maternal stress, sex and gestational age on laterality was assessed by adding maternal stress and a dummy factor for sex as explanatory variables to the binomial logit mixed model already described, testing the effect of each variable by omitting it from the model, as before. See Stroup (2013) for further details. Diagnostic tests were performed using profile zeta plots as described in Bates (2010), which assess the sensitivity of model fit to changes in the parameter estimates.

Analysis was carried out using the software environment R (R core team, 2013), using the lmer and glmer functions in the lme4 library (Bates et al, 2013). 


\section{Results}

Out of the 60 scans carried out, three scans were not of sufficient quality to allow accurate coding of the touching movements. Table 1 reports the number of left and right facial touches shown by each fetus at each gestational age. In total, we recorded 342 facial touches over 57 scans, 192 of which showed right laterality (56.1\%), a significant righthanded bias when compared to the null hypothesis of equal laterality proportions $\left(\chi^{2}=5.16(1 \mathrm{df}), \mathrm{p}=0.023\right)$. In terms of laterality preference for each fetus, eight fetuses showed right-handed preference, six showed left-handed preference and one fetus (F13) showed no preference.

We assessed within-fetus variability in laterality in two ways. Firstly, for each fetal scan, we calculated the dominant laterality (right, left or equal). We counted the number of switches between right, left and equal laterality over time. Only three of the 15 fetuses showed consistent laterality. The mean number of switches over the four gestational ages is 1.53 (std. dev. =1.06) showing considerable switching over the 13 week period. We also carried out a chi-squared test of equal proportions of right-handedness for each fetus over the four scans. These results were more inconclusive, with only four out of the 15 fetuses showing significant differences in proportion at the $\mathrm{p}=0.05$ level. Analyses of individual fetuses, however, lack power, and the mixed effects model using the combined data set provides a more powerful analysis. 


\section{Insert Table 1}

Maternal perceived stress score (PSS) ranged from 0 to 26, with a mean stress score over all scans of 12.05 (std. dev.= 7.09). Table 2 shows the result of the analysis of maternal stress by gestational age and sex of the fetus, using a linear mixed model. Both confidence intervals are consistent with regression parameters of zero, indicating that there is no significant trend of maternal stress over gestational age, and no fetal sex differences effect.

\section{Insert Table 2}

Table 3 and Figure 2 show the results of exploratory analyses examining the relationship between maternal perceived stress and handedness. Table 3 shows the individual stress scores for each fetus, divided into two groups: lower (PSS = 0-12) and higher (PSS=13-26). Shading is used to indicate whether there is right or left handed dominance when the results are disaggregated by high/low stress score. A pattern can be observed of left-handedness being associated with high stress and right-handedness with low stress.

Figure 2 shows the proportion of right-handed touches for each stress score, with a superimposed fitted logistic curve estimated through a binomial logit model. The slope of the logit curve is highly significant $\left(\chi^{2}=26.41(1), \mathrm{p}<0.001\right)$. A strong relationship can clearly be seen, with increasing stress being associated with decreasing right-handedness. However this exploratory analysis fails to take account of variation within and between fetuses. 
We now formally test the relationship between handedness, stress, fetal age and gender taking account of within and between fetal variation. Table 3 shows the results of the mixed binomial logit analysis. In looking at gestational age alone (Table 3a), no significant trend was observed, indicating that the proportion of right handed touches observed did not change significantly over gestational age. Although a slight positive increase in right handedness could be observed this was not significant $\left(\chi^{2}=0.890(1), \mathrm{p}=0.35\right)$.

Insert Table 3

Extending the above model, we examined whether the proportion of right-handed head touches could be related to perceived maternal stress level once sex and gestational age were controlled for (Table 3b). The effect of perceived maternal stress level over the previous four weeks was highly significant. The more stress mothers reported, the more frequently fetuses could be observed to touch their face with their left hand $\left(\chi^{2}=15.55(1), \mathrm{p}<0.0001\right)$. However, neither the sex of the fetus nor gestational age was associated with increasing righthandedness. The diagnostic profile zeta plots (Bates, 2010, p18) showed near-linearity, indicating that there were no concerns with the model estimates. The between-fetus variance estimate was 0.575 for the model including maternal stress indicating moderate residual variability between fetuses. Finally, in order to test whether stress reported for the last 4 weeks or earlier stress reported in the previous four to eight week period, we conducted a lag analysis, including lag of perceived stress level in the mixed binomial logit analysis. With a lagged analysis, we can only use three of the four scan times. A likelihood-ratio test on 
deleting the lag stress variable from this model showed that the lag stress variable was not significant $\left(\chi^{2}=2.56(1) ; \mathrm{p}=0.109\right)$.

\section{Discussion}

The present study suggests that maternal prenatal stress is associated with lateralised selftouch, with rising maternal perceived stress being linked with fetuses using increasingly their left hand to touch their face. This longitudinal study thus provides important new insights into the effect of maternal stress on the development of fetal predominant hand use for selftouch. The results indicate that maternal perceived stress experienced during the 4 weeks prior to the scan affected laterality rather than stress experienced in the 4-8 week period prior to the scan. Hence, current perceived stress seems more important in terms of lateralized behaviours observed. Our results show considerable variability in lateralised self-touch over the second and third trimester; 12 of the 15 fetuses switched the predominant hand for touching their face.

Research suggests that brain asymmetry and handedness are closely related (Toga \& Thompson, 2003) indicated by reliable differences in brain lateralization observed in left- and right-handers (Foundas, Corey, Hurley \& Heilman, 2006). Diffusion tensor imaging performed on fetal brains showed that cortical lateralization takes place between gestational weeks 15-26 in human fetuses (Gupta, Hasan, Trivedi, Pradhan, Das, Parikh et al, 2005). Potentially, this process of lateralization might be disturbed by maternal stress during pregnancy. There is some research indicating that high levels of prenatal maternal stress might be a risk factor for developmental disorders postnatally, since stress alters the biochemical equilibrium in the uterus (e.g.Van den Bergh, Mulder, Mennes \& Glover, 2005). Specifically, maternal stress during pregnancy results in increased levels of maternal cortisol, 
and is a potential mechanism for perturbing fetal brain development which might lead to altered laterality in the offspring (Glover et al , 2004).

Our findings indicate that hand preference for self-touch is not consistent within fetuses observed over the second and third trimester, nor does it seem to be related to gestational age. In our sample we found that maternal report of recent stress experienced during 4 weeks prior to the scan was significantly associated with fetal lateralized touch. Fetuses of stressed mothers showed proportionately more left handed self- touch compared with fetuses of mothers who reported relatively less stress experience in the previous 4 weeks. However, our study did not assess the stress level of the fetus, and so future studies should test fetal current cortisol levels in relation to lateralized self-touch.

There are some minor caveats to our work. A bias may have been introduced as some images only showed one hand. However, this was true for only $20 \%$, and such bias is thought to be minor. A second point is the measurement of stress using the PSS questionnaire. While respondents were asked to report on their stress levels in the four weeks prior to the scan, in practice it may be that some women just reported their current stress.

One measure of healthy development in infants is lateralized behaviour (Hall, Neal, \& Dean, 2008) including dominant hand use. Research indicates that the development of hand preference can be used as a biomarker for ill health including stress (Reissland, Hopkins, Helms, \& Williams, 2009) and psychopathy (van der Hooran, Oldehinkel, Ormel, Bruggeman, Uiterwaal, \& Burger, 2010). Mixed or left handedness is associated with developmental disorders, such as attention deficit-hyperactivity disorder and Autism Spectrum Disorders (Soper, Satz, Orsini, Henry, Zvi, \& Schulman, 1986). The results of the present study suggest that maternal perceived stress is significantly related to which hand the fetus uses to touch his or her head and face. Stressed mothers have fetuses who touch their 
Fetal laterality and maternal stress

face relatively more with their left hand. The research therefore highlights the importance of reducing maternal stress in pregnancy. Such measures may include increased emphasis on stopping stressful work early, the inclusion of relaxation classes in pre-natal care, and involvement of the whole family in the pre-natal period. Future research, therefore, also needs to examine whether maternal prenatally perceived stress affects infant and child development postnatally. 
Fetal laterality and maternal stress

\section{Acknowledgements}

We thank the mothers who took part in the study and Dr. Karen Lincoln for her support, as well as the independent coders of fetal facial movements. 


\section{References}

Bates, D. (2010) lme4: Mixed-effects modelling with R. Online book. Downloaded on 18 March 2014 from http://lme4.r-forge.r-project.org/lMMwR/lrgprt.pdf

Bates, D., Maechler. M., Bolker, B. and Walker, S. (2013). lme4: Linear mixed-effects models using Eigen and S4. R package version 1.0-5. http://CRAN.R-project.org/package=lme4

Cohen, S., Kamarck, T. \& Mermelstein, R. (1983). A global measure of perceived stress. Journal of Health and Social Behavior, 24, 386-396.

Cohen, S., \& Williamson, G. (1988). Perceived stress in a probability sample of the United States. In S. Spacapan \& S. Oskamp (Eds.), The social psychology of health: Claremont Symposium on applied social psychology. Newbury Park, CA: Sage.

de Vries, J. I. P., Wimmer, R.H., Ververs, I.A.P., Hopkins, B., Savelsbergh, G.J.P. \& van Geijn, H.P. (2001). Fetal handedness and head position preference: a developmental study. Developmental Psychobiology, 39, 171-178.

Dragovic, M., \& Hammond, G., (2005). Handedness in schizophrenia: a quantitative review of evidence. Acta Psychiatrica Scandinavia, 111, 410-419.

Fong, B.F., Buis, A.J., Savelsbergh, G.J., \& de Vries, J.I.(2005). Influence of breech presentation on the development of fetal arm posture. Early Human Development, 81, $519-527$.

Foundas, A.L., Corey, D.M., Hurley, M.M., \& Heilman, K.M.(2006). Verbal dichotic listening in right and left-handed adults: laterality effects of directed attention. Cortex, 42, 79-86. 
Glover, V., O'Connor, T.G., Heron, J., Golding, J., \& ALSPAC Study team (2004). Antenatal maternal anxiety is linked with atypical handedness in the child. Early Human Development , 79, 107-118.

Gupta, R.K., Hasan, K.M., Trivedi, R., Pradhan, M., Das, V., Parikh, N.A., \& Narayana, P.A. (2005). Diffusion tensor imaging of the developing human cerebrum. Journal of Neuroscience Research, 15, 172-178.

Hall, J.J., Neal, T.J., \& Dean, R.S.(2008). Lateralization of cerebral functions (3rd ed.). New York: Springer Publishing Company.

Heim. S., \& Keil, A. (2004). Large-scale neural correlates of developmental dyslexia. European Journal of Child and Adolescent Psychiatry, 13, 125-140.

Hepper, P.G., McCartney, G.R., Shannon, E.A. (1998) Lateralised behaviour in first trimester human foetuses. Neuropsychologia, 36, 531-534.

Hepper, P.G., Shahidullah, S., \& White, R. (1991). Handedness in the human fetus. Neuropsychologia, 29, 1107-1111.

Landau, R., Shusel, B., Eshel, Y. \& Ben Aaron, M. (2003). Mother-child and metapeletchild touch behavior with three-year-old kibbutz children in two contexts . Infant Mental Health Journal, 24, 529-546.

McManus, I.C. \& Bryden, M.P. (1991). Geschwind's Theory of Cerebral Lateralization: Developing a Formal, Causal Model. Psychological Bulletin, 110, 237-253.

Myowa-Yamakoshi, M., \& Takeshita, H.(2006). Do Human Fetuses Anticipate Self-Oriented Actions? A Study by Four-Dimensional (4D) Ultrasonography. Infancy, 10, 289-301. 
R Core Team (2013). R: A language and environment for statistical computing. R Foundation for Statistical Computing, Vienna, Austria. URL http://www.R-project.org/

Reissland, N., Hopkins, B., Helms, P., \& Williams, B. (2009). Maternal stress and depression and the lateralisation of infant cradling. Journal of Child Psychology and Psychiatry, 50, 263-269.

Snijders, T. A. B. \& Bosker, R.J. (2012) Multilevel Analysis: An introduction to basic and advanced multilevel modelling. New York: Wiley

Soper H.V., Satz, P., Orsini, D.L., Henry., R.R., Zvi, J.C., \& Schulman, M. (1986).

Handedness patterns in autism suggests subtypes. Journal of Autism and Developmental Disorders, 16, 155-167.

Sparling, J.W., van Tol, J., Chescheir, N.C. (1999). Fetal and neonatal handmovement. Physical Therapy, 79, 24-29.

Stroup, W.(2013). Generalized linear mixed models : modern concepts, methods and applications. Boca Raton : CRC Press.

Toga, A.W., \& Thompson, P.M. (2003). Mapping brain asymmetry. Natur Reviews Neuroscience, 4, 37-48.

Van den Bergh, B.R.H., Mulder, E.J.H., Mennes, M., \& Glover, V. (2005). Antenatal maternal anxiety and stress and the neurobehavioural development of the fetus and child: links and possible mechanisms. A review. Neuroscience and Biobehavioral Reviews, 29, 237-258. 
van der Hooran, A., Oldehinkel, A.J., Ormel, J., Bruggeman, R., Uiterwaal, C.S., \& Burger, H.(2010). Non-right-handedness and mental health problems among adolescents from the general population: The trails study. Laterality, 15, 304-316.

Ververs, I.A.P., van Gelder-Hasker, M.R., de Vries, J.I.P., Hopkins, B., \& van Geijn, H.P. (1998). Prenatal development of arm posture. Early Human Development, 51, 61-70.

Yang, J. J. , Han, H. ,Chui, D., Shen, Y., \& Wu, J.L. (2012). Prominent activation of the intraparietal and somatosensory areas during angle discrimination by intra-active touch. Human Brain Mapping, 33, 2957-2970. 
Table 1. Number of left and right facial touches for each fetal scan.

\begin{tabular}{|c|c|c|c|c|c|c|}
\hline \multirow[t]{2}{*}{$\begin{array}{l}\text { Sex and } \\
\text { Fetus ID }\end{array}$} & \multirow[t]{2}{*}{$\begin{array}{l}\text { Touch } \\
\text { laterality }\end{array}$} & \multicolumn{4}{|c|}{ Gestation } & $\begin{array}{l}\text { Number and } \\
\text { Percentage } \\
\text { over all scans }\end{array}$ \\
\hline & & 24 weeks & 28 weeks & 32 weeks & 36 weeks & N \% \\
\hline \multirow[t]{2}{*}{$\mathrm{F} 1$} & Left & 6 & 4 & 7 & 1 & $1878 \%$ \\
\hline & Right & 2 & 1 & 0 & 2 & $5 \quad 22 \%$ \\
\hline \multirow[t]{2}{*}{$\mathrm{M} 2$} & Left & 2 & & 0 & 4 & $6 \quad 67 \%$ \\
\hline & Right & 1 & & 0 & 2 & $333 \%$ \\
\hline \multirow[t]{2}{*}{ M3 } & Left & 1 & 1 & 4 & 9 & $1538 \%$ \\
\hline & Right & 11 & 3 & 0 & 10 & $24 \quad 62 \%$ \\
\hline \multirow[t]{2}{*}{ M4 } & Left & 2 & 0 & 4 & 0 & $633 \%$ \\
\hline & Right & 4 & 1 & 0 & 7 & $1267 \%$ \\
\hline \multirow[t]{2}{*}{ M5 } & Left & 7 & 0 & 2 & 6 & $1560 \%$ \\
\hline & Right & 7 & 1 & 2 & 0 & $10 \quad 40 \%$ \\
\hline \multirow[t]{2}{*}{ M6 } & Left & 0 & 1 & 1 & 1 & $3 \quad 18 \%$ \\
\hline & Right & 5 & 2 & 5 & 2 & $1482 \%$ \\
\hline \multirow[t]{2}{*}{$\mathrm{F7}$} & Left & 6 & 1 & 0 & 0 & $732 \%$ \\
\hline & Right & 0 & 2 & 8 & 5 & $15 \quad 68 \%$ \\
\hline \multirow[t]{2}{*}{ M8 } & Left & 3 & 1 & 3 & 3 & $10 \quad 52 \%$ \\
\hline & Right & 1 & 4 & 6 & 1 & $1248 \%$ \\
\hline \multirow[t]{2}{*}{ F9 } & Left & 1 & 1 & 4 & 0 & $6 \quad 40 \%$ \\
\hline & Right & 0 & 2 & 2 & 5 & $960 \%$ \\
\hline \multirow[t]{2}{*}{ F10 } & Left & & 4 & 1 & 2 & $7 \quad 18 \%$ \\
\hline & Right & & 11 & 11 & 9 & $3182 \%$ \\
\hline \multirow[t]{2}{*}{ M11 } & Left & 17 & 2 & 1 & 0 & $20 \quad 61 \%$ \\
\hline & Right & 4 & 4 & 2 & 3 & $1339 \%$ \\
\hline \multirow[t]{2}{*}{ F12 } & Left & 0 & 1 & 3 & & $4 \quad 27 \%$ \\
\hline & Right & 4 & 4 & 3 & & $1173 \%$ \\
\hline \multirow[t]{2}{*}{$\mathrm{F} 13$} & Left & 2 & 3 & 1 & 0 & $\begin{array}{ll}6 & 50 \% \\
\end{array}$ \\
\hline & Right & 1 & 4 & 3 & 3 & $1150 \%$ \\
\hline \multirow[t]{2}{*}{ F14 } & Left & 2 & 4 & 2 & 4 & $1256 \%$ \\
\hline & Right & 2 & 2 & 3 & 2 & $944 \%$ \\
\hline \multirow[t]{2}{*}{ F15 } & Left & 2 & 2 & 3 & 2 & $938 \%$ \\
\hline & Right & 6 & 6 & 0 & 3 & $1562 \%$ \\
\hline
\end{tabular}

Note: Three scans (one for male fetus M2 and two for female fetusus F10 and F12) did not demonstrate the stringent conditions for touching during the 600 second scan. 
Table 2. Results of linear mixed effects (multi-level) modelling of self-reported maternal stress by fetal age and sex .

\begin{tabular}{|c|c|c|}
\hline & $\begin{array}{l}\text { Estimate } \\
\text { b }\end{array}$ & 95\% Cl for $\mathbf{6}$ \\
\hline$\beta_{0}$ (intercept) & 17.094 & $(8.22,25.96)$ \\
\hline$\beta_{1}$ (gestational age) & -0.111 & $(-0.368,0.146)$ \\
\hline$\beta_{2}$ (sex =female) & -3.58 & $(-9.942,2.732)$ \\
\hline$\sigma_{\text {fetus }}^{2}$ & 29.39 & $(12.92,72.44)$ \\
\hline$\sigma_{e}^{2}$ & 18.17 & $(12.18,28.81)$ \\
\hline
\end{tabular}


Table 3. Results of the binomial logit mixed effects modelling of the proportion of right handed head touches a) by gestational age and b) by self-reported maternal stress, gestational age and sex

\begin{tabular}{|c|c|c|c|c|}
\hline & Estimate $\beta$ & $\begin{array}{l}\text { Odds ratio } \\
\operatorname{Exp}(\beta)\end{array}$ & $\begin{array}{l}95 \% \mathrm{Cl} \text { for odds } \\
\text { ratio }\end{array}$ & LRT \\
\hline \multicolumn{5}{|c|}{ a) effect of gestational age alone } \\
\hline$\beta_{0}$ (intercept) & -0.461 & 0.631 & $(0.137,2.929)$ & \\
\hline$\beta_{1}$ (gestational age) & 0.024 & 1.024 & $(0.975,1.075)$ & $\begin{array}{l}0.890 \text { on } 1 \mathrm{df} ; \\
\mathrm{p}=0.35\end{array}$ \\
\hline$\sigma_{\text {fetus }}^{2}$ & 0.341 & & $(0.083,1.069)$ & \\
\hline \multicolumn{5}{|c|}{ b) Effect of gestational age, maternal stress and sex } \\
\hline$\beta_{0}$ (intercept) & 1.111 & 3.037 & $(0.592,15.995)$ & \\
\hline$\beta_{1}$ (gestational age) & 0.010 & 1.010 & $(0.962,1.060)$ & $\begin{array}{l}0.163 \text { on } 1 \mathrm{df} ; \\
\mathrm{p}=0.69\end{array}$ \\
\hline$\beta_{2}$ (maternal stress) & -0.089 & 0.915 & $(0.883,0.948)$ & $\begin{array}{l}15.55 \text { on } 1 d f ; \\
p<0.0001\end{array}$ \\
\hline$\beta_{3}$ (sex =female) & -0.104 & 0.901 & $(0.566,1.435)$ & $\begin{array}{l}0.193 \text { on } 1 \mathrm{df} ; \\
p=0.66\end{array}$ \\
\hline$\sigma_{\text {fetus }}^{2}$ & 0.575 & & $(0.309,0.841)$ & \\
\hline
\end{tabular}


Fetal laterality and maternal stress

\section{Figure captions}

Figure 1. Fetal left hand touching the upper face. Fingers and wrist are visible, thumb is turned into palm. Appearance of the thickness of the fingers and appearance caused by the thumb being folded into the palm are both used to identify left or right hand.

Figure 2. The proportion of right-handed touches for each stress score, with fitted curve from logistic regression. 